\section{Study of biometric parameters in family members of primary angle closure glaucoma patients}

R Sihota1', D Ghate1, S Mohan', V Gupta1,

R Sihota ${ }^{1}$, D Ghate', S Moh
RM Pandey ${ }^{2}$ and T Dada ${ }^{1}$

\section{Introduction}

Eyes having primary angle closure glaucoma (PACG) are known to have a shorter axial length $(\mathrm{AL})$, shallower anterior chambers $(\mathrm{AC})$, and thicker lens. ${ }^{1-4}$ Certain racial groups are at an increased risk of developing PACG. ${ }^{5-9} \mathrm{~A}$ positive family history of PACG is an additional risk factor. The inheritance of PACG is believed to be polygenic, ${ }^{10-13}$ although both autosomal dominant and recessive inheritance pattern are seen in pedigrees with a high prevalence of PACG. The importance of anatomical factors in PACG has evoked considerable curiosity as to whether they are genetically determined. A similarity between these dimensions in patients and their relatives may explain their predisposition of acquiring the condition and conversely any dissimilarity may explain why many relatives do not become affected. Thus, the role of ocular dimensions needs further evaluation in relatives of PACG patients. The aim of this study was to measure ocular biometric parameters in untreated family members of index PACG patients, to compare these values in affected, unaffected, and suspected family members and to determine the factors that could be predictive of angle closure in family members of PACG patients.

\section{Patients and methodology}

Consecutive patients of PACG attending a tertiary care center were included in the study. Informed consent was obtained from all index patients and their family members and the study was approved by the institutes review board. All possible first-and second-degree relatives of the index patients more than 20 years of age were screened for glaucoma. These included family members who
Keywords: primary angle closure; biometry; family members; lens thickness; axial length
${ }^{1}$ Glaucoma Research Facility \& Clinical Services, $\mathrm{Dr}$ Rajendra Prasad Centre for Ophthalmic Sciences, All India Institute of Medical Sciences, New Delhi, India

${ }^{2}$ Department of Biostatistics, All India Institute of Medical Sciences, New Delhi, India

Correspondence: V Gupta, Assistant Professor of Ophthalmology,

Dr Rajendra Prasad Centre for Ophthalmic Sciences, All India Institute of Medical Sciences,

Ansari Nagar,

New Delhi-110029, India

Tel: + 911126862527 ;

Fax: + 911126588919

E-mail: gupta_v20032000@ yahoo.com

Received: 1 June 2006 Accepted in revised form: 1 October 2006

Published online:

16 February 2007

Disclosure: None of the authors have any financial or proprietary interest in the subject matter of presentation. 
accompanied the index case and those members called to the hospital by writing to them from the addresses given by the index patient. Families where three or more members were available for screening were included in the study and the rest were excluded. A detailed history and complete examination including best-corrected visual acuity, slit-lamp biomicroscopy, fundus examination, applanation tonometry, indentation gonioscopy, perimetry with Humphrey's visual field analyzer using 30-2 full threshold strategy, and optic disc evaluation using HRT II was performed by a glaucomatologist.

Biometric parameters analyzed were anterior chamber depth (ACD), AL, lens thickness (LT), and central corneal thickness (CCT), which were measured on a Sonomed PacScan 333AP machine (LPO, OH , USA). The corrected ACD was calculated by subtracting CCT from ultrasonically measured ACD. Relative lens position (LP) was calculated by the formula $\mathrm{LP}=([\mathrm{ACD}+0.5 \mathrm{LT}] / \mathrm{AL}){ }^{8}$ Both eyes of each family member were examined and if one eye was found affected, the member was labeled as being affected, and biometric parameters of that eye were used for analysis. If both eyes of a member were affected, the right eye was taken for analysis. All patients were worked up by one observer (optometrist) who was masked to the clinical classification of the patient.

The family members were divided into affected, suspected, and unaffected groups. Affected family members were categorized into various subtypes of glaucoma based on the following definitions.

\section{Affected family members}

\section{(a) Primary angle closure}

Gonioscopically documented presence of peripheral anterior synechiae or clumping of pigment in an occludable angle, that is, one with a steep peripheral iris configuration. There may or may not have been a history of unilateral headaches, blurring of vision or colored haloes occurring periodically, and resolving spontaneously.

\section{(b) Acute primary angle closure}

The occurrence of an acute attack of angle closure with severe unilateral headache, diminution of vision-associated nausea/vomiting, and the presence of an IOP $>22 \mathrm{~mm} \mathrm{Hg}$ in an eye with a shallow anterior chamber conjunctival injection, corneal edema and mid-dilated pupil without glaucomatous optic neuropathy.

\section{(c) Chronic primary angle closure glaucoma}

Eyes that had a chronically (on at least three separate occasions) elevated IOP ( $>21 \mathrm{~mm} \mathrm{Hg}$ ), gonioscopically confirmed peripheral anterior synechiae of more than $180^{\circ}$, and optic nerve head and/or visual field changes.

PAC suspects were those with occludable angles. An occludable angle was diagnosed when an eye had more than $180^{\circ}$ of posterior trabecular meshwork not visible gonioscopically without manipulation or indentation.

\section{Unaffected}

Family members having gonioscopically open angles (angle recess $>25^{\circ}$ ), with no evidence of POAG/PAC suspect/PACG.

Cases having any other ocular disease, prior laser iridotomy, prior surgery like cataract surgery, trabeculectomy, lasik, etc, were excluded from the study. A relative was considered to be first degree if he/she was either a parent, child or sibling of the index case whereas a second-degree relative was one who was genetically two meiosis away from the index case in the family tree.

\section{Statistical analysis}

To compare various biometric parameters among index, affected, suspected, and unaffected family member, we considered each family as the cluster. It was anticipated that within family, the biometric parameters of index case, affected case, suspected, and unaffected family member are likely to be correlated. Therefore, we treated the index, affected, suspected, and unaffected groups as correlated, and analyzed the correlated data using the generalized estimating equation regression approach. The difference of the biometric parameters from the index patients was calculated and the overall averages were analyzed. ROC curve was plotted for the biometric parameters to determine the cutoff values and their ability to predict PAC cases (index + affected) as compared with nonPAC cases (suspects + unaffected). Correlation between various parameters was calculated by Spearman's correlation coefficient. Statistical analysis was performed using STATA 8.0 version software (STATA Corporatation, College Station Road, Houston, TX, USA). In this study, $P$-values less than 0.05 were considered as statistically significant.

\section{Results}

In the study, 28 families of 28 index patients were included from a hospital-based sample. All patients were Asians of Indian ethnicity. There were 108 family members (215 eyes) fulfilling our inclusion criterion. On 
an average, 3.8 members per index case were seen. A minimum of three and a maximum of 13 family members were screened. Eighty-four were first-degree relatives whereas 24 were second-degree relatives.

Thirty-four (31.6\%) members were found to be affected family members, $19(17.6 \%)$ were diagnosed to be suspect, and $55(50.7 \%)$ were unaffected family members. Thirty $(35.3 \%)$ of the first-degree members and four $(16.7 \%)$ of the second-degree members were found to be affected by primary angle closure . Similarly, 16 (19\%) among the first-degree relatives and six (12.5\%) among the second degree were PAC suspects, whereas 38 $(45.2 \%)$ and $17(70 \%)$, respectively, were unaffected.

The mean age of index patients $(55.53 \pm 12.9$ years) was significantly higher than the affected, suspected, unaffected family members and from the mean age of the first-degree and second-degree family members (Table 1). In the affected members, the male: female ratio was $1: 1.4$, among suspects $1: 0.7$, and among unaffected $1: 0.9$.

The range of the spherical correction was $-0.19 \mathrm{D}$ to $+0.93 \mathrm{D}$ for affected, $-1.24 \mathrm{D}$ to $+0.53 \mathrm{D}$ for the suspects, and $-0.16 \mathrm{D}$ to $+0.90 \mathrm{D}$ for the unaffected family members.

The biometric parameters are shown in Table 2. Mean ACD was significantly $(P=0.0001)$ shallower in affected family members $(2.29 \pm 0.35 \mathrm{~mm})$ compared with unaffected members $(2.83 \pm 0.15 \mathrm{~mm})$. The unaffected eyes had deeper ACD and thinner lens than the other groups.

The comparative evaluation of the mean value of LT among family members for affected $(4.04 \pm 0.45 \mathrm{~mm})$, suspected $(4.02 \pm 0.43 \mathrm{~mm})$ and unaffected

$(3.82 \pm 0.25 \mathrm{~mm})$ was not significant statistically (Table 2$)$. The mean value for the lens position was same for the all groups of family members and in the first-degree and second-degree relatives (Tables 2-4).

The AL values between affected $(22.58 \pm 1.2 \mathrm{~mm})$ and unaffected family members $(23.74 \pm 1.36 \mathrm{~mm})$ was significantly different $(P=0.021)$ (Table 2$)$. The AL in affected $(22.58 \pm 1.03 \mathrm{~mm})$ and suspected

$(23.21 \pm 0.73 \mathrm{~mm})$ family members showed no significant difference $(P=0.117)$. There was also no significant difference in AL between suspects and unaffected eyes.

The percentage mean difference of the biometric parameters from the index patients were calculated (Figure 1). ACD was $14.56 \%$ more in affected, $21.7 \%$ more in suspected, and $34.92 \%$ in unaffected family members. Similarly, LT was $10.73,11.1$, and $16.08 \%$ less and AL was $0.11,3.53$, and $5.37 \%$ more in affected, suspected, and unaffected family members compared to index cases.

Biometric parameters were also compared between the index and first-degree and second-degree relatives (Table 3). The ACD and corrected ACD showed lower values for the index $v$ first-degree $(P=0.0001)$ and firstdegree $v$ s second-degree family members $(P=0.0001)$.

The AL was shorter in index cases compared with other family members. LT was more in first-degree relatives. Lens position was same for both first- and second-degree family members (Tables 3 and 4).

The biometric parameters were also analyzed among the first- and second-degree relatives. In first-degree relatives, the lens was thicker and ACD shallower among affected and PAC suspects compared with the unaffected members. The AL was significantly longer in unaffected compared to the affected members. There were fewer second-degree relatives but even among those the ACD and AL were significantly different in affected and unaffected eyes.

There was sufficient correlation of age with ACD and corrected ACD in affected first-degree family members (Table 5). Spearman's correlation coefficient calculated between corrected ACD and LT was found to be significant $(r=-0.532, P=0.01)$. The ROC curve plotted for biometric parameters in the family members are shown in Figure 2. Cutoff value for CACD was found to be $2.24 \mathrm{~mm}$, for which sensitivity was $86.3 \%$ and specificity was $50.47 \%$. Similarly, cutoff value for LT was

Table 1 Demographic characteristics of PACG patients and their family members

\begin{tabular}{llcrr}
\hline Groups & Degree of family members & Age (years) Mean $\pm S D$ & Overall mean age years $( \pm S D)$ & Male:female ratio \\
\hline Index $(n=28)$ & & & $55.53 \pm 12.9^{\mathrm{a}, \mathrm{b}, \mathrm{c}}$ & $1: 1.5$ \\
Affected $(n=34)$ & First-degree & $45.0 \pm 13.3$ & $49.375( \pm 14.4)^{\mathrm{b}, \mathrm{c}, \mathrm{d}}$ & $1: 1.4$ \\
& Second-degree & $51 \pm 12.0$ & $37.19( \pm 10.5)^{\mathrm{a}, \mathrm{c}, \mathrm{d}}$ & $1: 0.7$ \\
Suspected $(n=19)$ & First-degree & $40.9( \pm 8.5)$ & $36.00( \pm 13.1)^{\mathrm{a}, \mathrm{b}, \mathrm{d}}$ & $1: 0.9$ \\
& Second-degree & $38.8( \pm 5.5)$ & &
\end{tabular}

Abbreviations: PACG, primary angle closure glaucoma; SD, standard deviation.

${ }^{\text {a }} P<0.05$ in relation to Affected.

${ }^{\mathrm{b}} P<0.05$ in relation to Suspect.

${ }^{c} P<0.05$ in relation to Unaffected.

${ }^{\mathrm{d}} P<0.05$ in relation to Index. 
Table 2 Mean (95\% CI) of biometric parameters in index, affected, suspected, and unaffected family members, using generalized estimating equation (GEE) regression

\begin{tabular}{llcccc}
\hline & \multicolumn{1}{c}{ Groups } & Anterior chamber depth $(\mathrm{mm})$ & Lens Thickness $(\mathrm{mm})$ & Axial length $(\mathrm{mm})$ & Central corneal thickness $(\mu \mathrm{m})$ \\
\hline 1 & Index $(\mathrm{N}=28)$ & $2.59(2.42-2.76)$ & $4.66(4.46-4.86)$ & $22.55(22.07-23.01)$ & $547.89(530.95-564.83)$ \\
2 & Affected $(N=34)$ & $2.87(2.68-3.06)$ & $4.04(3.75-4.33)$ & $22.58(22-23.09)$ & $542.51(506.14-578.88)$ \\
3 & Suspects $(\mathrm{v}=19)$ & $3.06(2.89-3.22)$ & $4(3.84-4.19)$ & $23.21(22.5-23.81)$ & $560.98(537.79-584.17)$ \\
4 & Unaffected (N=55) & $3.4(3.33-3.47)$ & $3.82(3.67-3.95)$ & $23.74(22.7-24.73)$ & $557.79(539.15-576.43)$ \\
5 & Overall $P$-value & $t=48.45, P=0.0001$ & $t=42.45, P=0.0001$ & $t=71.0, P=0.0001$ & $t=54.11, P=0.001$ \\
6 & $P$-value I vs II & $t=2.38, P=0.025$ & $t=4.32, P=0.0001$ & $t=0.1, P=0.92$ & $t=0.27, P=0.786$ \\
7 & $P$-value I vs III & $t=4.35, P=0.0001$ & $t=5.14, P=0.0001$ & $t=1.9, P=0.068$ & $t=1.04, P=0.305$ \\
8 & $P$-value I vs IV & $t=9.47, P=0.0001$ & $t=8.87, P=0.0001$ & $t=2.44, P=0.02$ & $t=0.93, P=0.361$ \\
9 & $P$-value II vs III & $t=1.64, P=0.113$ & $t=0.17, P=0.863$ & $t=1.62, P=0.117$ & $t=0.74, P=0.467$ \\
10 & $P$-value II vs IV & $t=4.96, P=0.0001$ & $t=1.46, P=0.155$ & $t=2.45, P=0.021$ & $t=0.78, P=0.440$ \\
11 & $P$-value III vs IV & $t=3.93, P=0.001$ & $t=1.92, P=0.065$ & $t=0.97, P=0.339$ & $t=0.27, P=0.789$ \\
\hline
\end{tabular}

Table 3 Comparison of mean (SD) of biometric parameters in first and second-degree relatives, using generalized estimating equation (GEE) regression

\begin{tabular}{lccc}
\hline Biometric parameters & Index $(\mathrm{n}=28)$ & First-Degree family members $(\mathrm{n}=84)$ & Second-degree Family members $(\mathrm{n}=24)$ \\
\hline ACD $(\mathrm{mm})$ & $2.58(0.41)$ & $3.08(0.40)^{\mathrm{a}}$ & $3.33(0.32)^{\mathrm{a}, \mathrm{b}}$ \\
CACD $(\mathrm{mm})$ & $2.03(0.41)$ & $2.52(0.41)^{\mathrm{a}}$ & $2.78(0.32)^{\mathrm{a}, \mathrm{b}}$ \\
LT $(\mathrm{mm})$ & $4.64(0.53)$ & $4.02(0.61)^{\mathrm{a}}$ & $3.79(0.43)^{\mathrm{a}, \mathrm{b}}$ \\
LP $(\mathrm{mm})$ & $0.22(0.02)$ & $0.22(0.02)$ & $0.22(0.02)$ \\
AL $(\mathrm{mm})$ & $22.48(1.15)$ & $23.19(1.61)^{\mathrm{a}}$ & $23.30(1.93)^{\mathrm{a}}$ \\
CCT $(\mathrm{mm})$ & $0.547(0.04)$ & $0.559(0.04)$ & $0.559(0.04)$ \\
\hline
\end{tabular}

${ }^{a} P<0.05$ in comparison to the index.

${ }^{\mathrm{b}} P<0.05$ in comparison to the first degree.

Table 4 Comparision of Mean (SD) of biometric parameters in affected, suspected, and unaffected within first and second-degree family members: using generalized estimating equation (GEE) regression

\begin{tabular}{|c|c|c|c|c|c|c|c|c|}
\hline \multirow[t]{2}{*}{ Biometric parameters $(\mathrm{mm})$} & \multicolumn{4}{|c|}{ First-degree family members $(\mathrm{n}=84)$} & \multicolumn{4}{|c|}{ Second-degree family members $(\mathrm{n}=24)$} \\
\hline & $\begin{array}{l}\text { Affected } \\
(\mathrm{n}=30)\end{array}$ & $\begin{array}{l}\text { Suspects } \\
(\mathrm{n}=16)\end{array}$ & $\begin{array}{l}\text { Unaffected } \\
(\mathrm{n}=38)\end{array}$ & $\begin{array}{l}\text { Overall } \\
\text { P-value }\end{array}$ & $\begin{array}{c}\text { Affected } \\
(\mathrm{n}=4)\end{array}$ & $\begin{array}{c}\text { Suspects } \\
(\mathrm{n}=3)\end{array}$ & $\begin{array}{l}\text { Unaffected } \\
(\mathrm{n}=17)\end{array}$ & $\begin{array}{l}\text { Overall } \\
\text { P-value }\end{array}$ \\
\hline ACD & $2.8(0.5)$ & $2.8(0.3)$ & $3.3(0.3)^{a, b}$ & 0.0001 & $3.1(0.1)$ & $3.4(0.3)$ & $3.4(0.3)^{\mathrm{a}}$ & 0.0482 \\
\hline CACD & $2.2(0.5)$ & $2.2(0.3)$ & $2.8(0.3)^{a, b}$ & 0.0001 & $2.5(0.1)$ & $2.8(0.2)$ & $2.8(0.3)^{\mathrm{a}}$ & 0.0704 \\
\hline LT & $4.1(0.8)$ & $4.2(0.4)$ & $3.8(0.5)^{a, b}$ & 0.003 & $3.80(0.4)$ & $3.8(0.3)$ & $3.7(0.3)$ & 0.0762 \\
\hline LP & $0.21(0.02)$ & $0.21(0.02)$ & $0.22(0.02)$ & 0.9557 & $0.22(0.02)$ & $0.23(0.01)$ & $0.22(0.02)$ & 0.988 \\
\hline $\mathrm{AL}$ & $22.6(1.2)$ & $22.8(2.1)$ & $23.6(1.7)^{\mathrm{a}}$ & 0.0038 & $22.5(1.2)$ & $22.9(0.6)$ & $23.6(2.3)^{\mathrm{a}}$ & 0.03226 \\
\hline ССТ & $0.561(0.04)$ & $0.564(0.05)$ & $0.556(0.04)$ & 0.706 & $0.553(0.02)$ & $0.553(0.02)$ & $0.561(0.05)$ & 0.839 \\
\hline
\end{tabular}

Abbreviations: ACD, anterior chamber depth; AL, axial length; $\mathrm{CACD}$, corrected anterior chamber depth; CCT, central corneal thickness; LT, lens thickness.

${ }^{\text {a }} P<0.05$ in comparison to the affected.

${ }^{\mathrm{b}} P<0.05$ in comparison to the suspects.

calculated to be $4.28 \mathrm{~mm}$ with $81.25 \%$ sensitivity and $58.4 \%$ specificity, and for the AL it was found to be $21.8 \mathrm{~mm}$ with a sensitivity of $74 \%$ and a specificity of $59 \%$.

\section{Discussion}

Surveys have shown a prevalence of PACG of $1.08-4.32 \%^{13-15}$ in individuals over 40 years of age. The incidence of occludable angles in first-degree relatives of patients with PACG has been reported to be 3.5-6 times higher ${ }^{10}$ than that of the general population. In our study among Asians of Indian ethnicity family members had an 8-9 times higher incidence of primary angle closure. The prevalence of PACG among firstdegree relatives in the Caucasian population is variously given as $1-12 \% .{ }^{16}$ Spaeth $^{17}$ estimated the frequency of occludable angles among first-degree relatives of Caucasian PACG probands at $20 \%$, four to five times higher than that for the population at large. 
To the best of our knowledge, there are no studies comparing the biometric data among PACG patients and their first-degree and second-degree family members to determine those at risk. The strength of this study lies in the fact that all possible family members were screened and biometry performed before instituting any therapy, which could have altered the parameters. Our study agrees well with findings of other workers ${ }^{13,18-22}$ regarding PACG index patients, who had significantly shallow anterior chamber depths, thicker lenses and shorter ALs than their family members. Although increasing age seems to be a significant confounding factor among the affected patients as compared with unaffected members, affected family members were seen in both first- and second-degree relatives of all ages.

Precipitation of an attack of angle closure glaucoma depends on various circumstances, but the most important factors are undoubtedly a shallow anterior chamber and an occludable angle. The presence of shallower anterior chambers in relatives of index PACG patients, as in this study, has also been reported by Tomlinson et al. ${ }^{8}$ In this study, we were able to show a biometric gradation of ACD, lens thickness LT and AL in affected, suspect, and normal eyes of family members. The ACD and LT values were also significantly different between eyes of index, first-degree, and second-degree

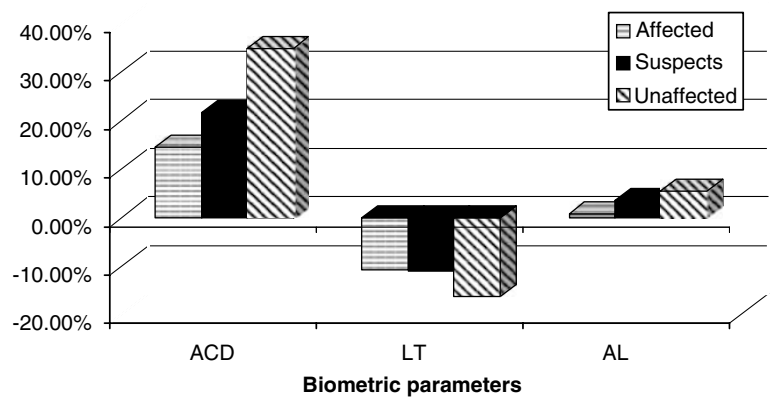

Figure 1 Percentage value of difference of biometric parameters for affected, suspects, and unaffected members in relation to index patients. family members. Among first-degree relatives and second-degree relatives, the lens was thicker and the ACD was shallower in affected family members, as compared with those unaffected within these subgroups. Alsbirk ${ }^{18}$ also found age-and sex-independent shallowness of ACD among relatives of PACG probands, suggesting a hereditary component.

Increasing LT with age may confound attempts to use this parameter and also ACD as a measure of identifying family members at risk of developing PAC. As AL growth stabilizes at 18 years, a comparison of AL measurements could be used as a marker for possible PAC. The mean AL for index patients in our study was $22.55 \mathrm{~mm}$, corroborating well with the study reported by George et al. ${ }^{22}$ The AL for unaffected family members was greater across the subgroups, in both first, and second-degree relatives compared with affected family members.

In our study, relative lens position was the same in all the groups of family members.

As the relative lens position was similar in all family members studied by us, the occurrence of PAC in our families appears to be largely related to a shorter AL and greater LT and their effect on anterior chamber depth. Lowe $^{6}$ in his study postulated that with normal ocular development, anteriorly placed, thicker lens are found in shorter eyes; therefore, a slight incoordination would result in PACG. For a thick lens to be anteriorly sited in a long eye, a gross incoordination would be necessary, a rare occurrence. Tomlinson and Leighton ${ }^{8}$ studied ocular dimensions in index patients with angle closure glaucoma and a few first-degree relatives, siblings, and offspring. Their index patients had already undergone iridotomy, which is known to change ACD and lens position measurements. Index patients, siblings, and offspring were all found to have a shallower ACD and a thicker lens as compared with normals. Their comparison of patients with PACG and normal subjects showed that the lens was significantly more anterior in patients than controls, but was similarly sited in siblings of patients and controls. Their study also showed that the relative lens position shows less significant differences

Table 5 Spearmann's rank correlation coefficient for the biometric parameters with age

\begin{tabular}{|c|c|c|c|c|c|c|}
\hline \multirow[t]{2}{*}{ Biometric parameters } & \multicolumn{3}{|c|}{ First-degree family members $(\mathrm{n}=84)$} & \multicolumn{3}{|c|}{ Second-degree family members $(\mathrm{n}=24)$} \\
\hline & Affected $(\mathrm{n}=30)$ & Suspects $(\mathrm{n}=16)$ & Unaffected $(\mathrm{n}=38)$ & Affected $(\mathrm{n}=4)$ & Suspects $(\mathrm{n}=3)$ & Unaffected $(\mathrm{n}=17)$ \\
\hline $\mathrm{ACD}$ & $-0.52^{*}$ & -0.09 & -0.12 & -0.87 & -0.99 & -0.07 \\
\hline CACD & $-0.52^{*}$ & -0.09 & -0.08 & -1.0 & -0.99 & -0.07 \\
\hline LT & +0.28 & -0.12 & +0.24 & -0.94 & +0.91 & +0.37 \\
\hline $\mathrm{AL}$ & -0.23 & +0.73 & +0.02 & +0.12 & +0.05 & +0.24 \\
\hline ССТ & -0.09 & -0.08 & -0.24 & +0.52 & +0.99 & +0.04 \\
\hline
\end{tabular}

Abbreviations: ACD, anterior chamber depth; CCT, central corneal thickness; LT, lens thickness; AL, axial length.

${ }^{*} P<0.05$. 

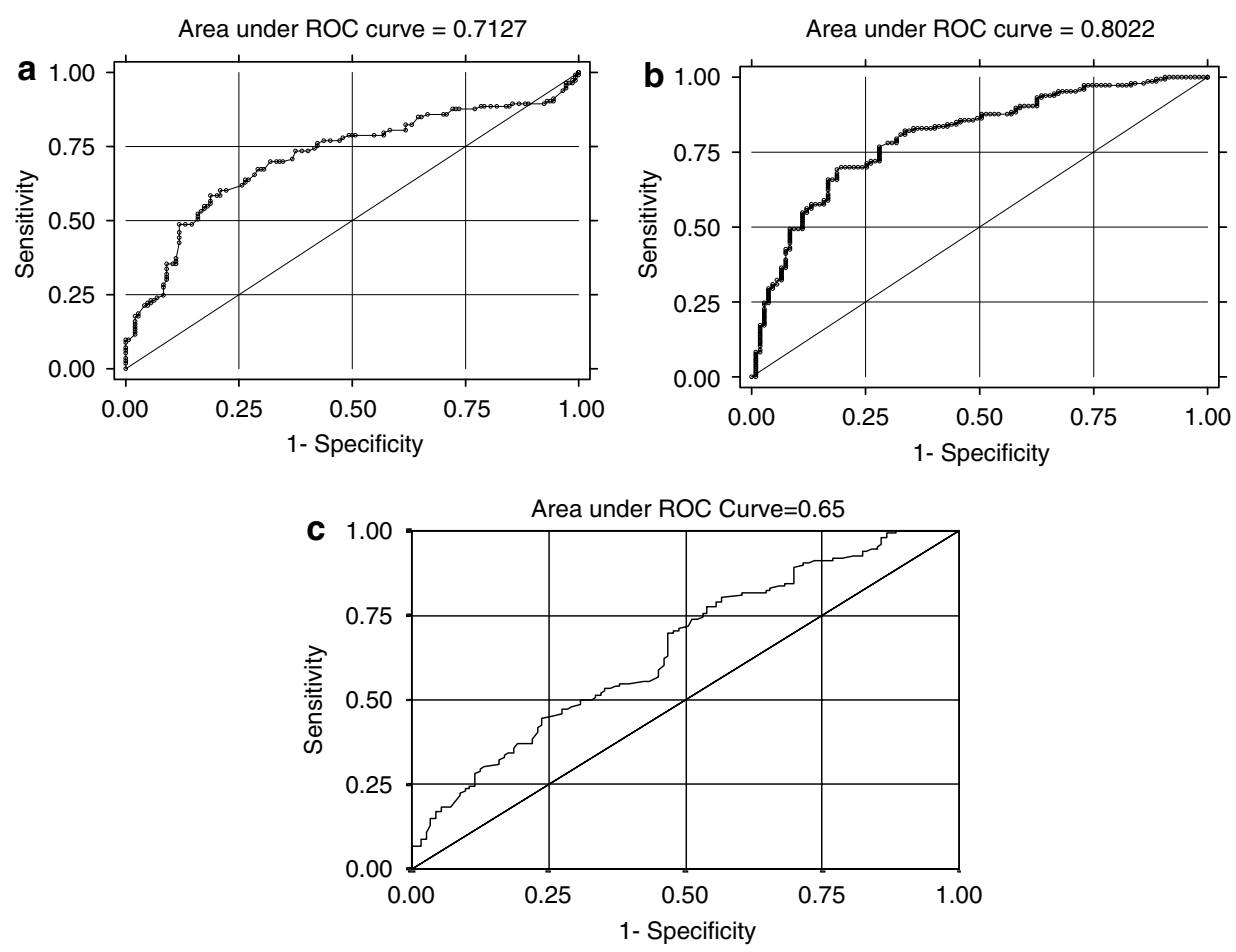

Figure 2 ROC of LT (a), corrected ACD (b), and AL (c) in the index and affected $v$ s suspect and unaffected cases.

between patients and their unaffected sibling than did ACD, which is also clear from our study.

Analyzing the various parameters of eyes having PACG, PAC suspects, and unaffected family members, it was seen that a corrected ACD of $2.24 \mathrm{~mm}$ or less and a LT of $4.28 \mathrm{~mm}$ or more had a fairly good sensitivity for the diagnosis of primary angle closure in family members. Within each family cluster, it was seen that an increase in ACD of about 35\%, a decrease in LT of $16 \%$, and an AL longer by 5\% compared with the index cases seem to identify those family members with an open angle on gonioscopy. These biometric parameters can thus be used to identify younger family members of PACG patients who are at risk.

Many patients with shallow anterior chambers have been found to be hypermetropic; therefore, many authors inferred that angle closure glaucoma occurred in small hypermetropic eyes. ${ }^{20}$ In our study, the refractive error calculated did not show any significant difference in the groups and the mean values were less than $+1.0 \mathrm{D}$. But Grieten and Weekers ${ }^{23}$ showed that eyes with angle closure glaucoma had anterior chambers $0.73 \mathrm{~mm}$ more shallow, corneal curvatures $0.20 \mathrm{~mm}$ less, and corneal diameters $0.48 \mathrm{~mm}$ smaller than eyes of the same age with the same degree of hypermetropia. Lowe ${ }^{4}$ noted that eyes with PACG are mostly hypermetropic; nevertheless, some are also myopic, so that hypermetropia is insufficient to explain the anatomical conditions that lead to angle closure glaucoma.
Our study had limitations of a relatively small sample size. Also as it was not a population-based study and so remains exposed to the biases of a hospital-based study. Age and sex were not matched for the groups studied because of limited number of members in a family available for examination. Certain members had to be excluded because of prior laser procedures, surgery, or other ocular diseases. Our study was a cross-sectional study, and a longitudinal study based on repeated measurements over a number of years would help increase the predictability of these findings.

To conclude, the incidence of primary angle closure in family members of PACG is higher than reported previously. The ACD is narrowest, lens is thickest and AL shortest in family members having PACG and these gradually move towards normal values in suspected and unaffected family members. Knowing the biometric parameters of an index patient, especially the AL, which does not change with age, iridotomy, or surgery, could help in the early identification of family members at risk for primary angle closure glaucoma.

\section{References}

1 Sihota R, Agarwal H C. Profile of the subtypes of angle closure glaucoma in a tertiary hospital in India. Ind $J$ Ophthalmol 1998; 46: 25-29.

2 Sihota R, Gupta V, Pandey RM, Kumar D, Agarwal HC. A comparison of symptomatic and asymptomatic chronic 
primary angle closure glaucoma. J Glaucoma 2000; 9: 208-213.

3 Sihota R, Lakshmaiah NC, Pandey RM, Agarwal HC. Ocular parameters in the subtypes of primary angle closure glaucoma. Clin Exp Ophthalmol 2000; 28: 253-258.

4 Lowe RF. Clinical types of primary angle closure glaucoma. Aust NZ J Ophthalmol 1988; 16: 245-250.

5 Gupta S, Sood NN, Dayal Y. Angle closure glaucoma IV. East Arch Ophthalmol 1975; 3: 238-242.

6 Lowe RF. Aetiology of the anatomical basis for primary angle closure glaucoma. Biometrical comparisons between normal eyes and eyes with primary angle closure glaucoma. Br J Ophthalmol 1970; 54: 161-169.

7 Philips CI, Storey JK. Ocular dimensions in angle closure glaucoma. Br J Physiol Optics 1971; 26: 228-242.

8 Tomlinson A, Leighton DA. Ocular dimensions in the heredity of angle closure glaucoma. Br J Ophthalmol 1973; 57: 475-486.

9 Sood NN, Jain RC, Agarwal HC. Ocular biometry in primary angle closure glaucoma in Indians. Ind J Med Res 1988; 88: 190-191.

10 Wilensky JT, Kaufman PL, Frohlichstein D, Gieser DK, Kass MA, Ritch R et al. Follow up of angle closure glaucoma suspects. Am J Ophthalmol 1993; 115: 335-345.

11 Alsbrik PH. Anterior chamber depth, gene and environment. Acta Ophthalmol 1982; 60: 223-234.

12 Lowe RF. Primary angle closure glaucoma: Inheritence and environment. Br J Ophthalmol 1972; 56: 13-20.

13 Torniquist R. Chamber depth in primary angle closure glaucoma. Br J Ophthalmol 1956; 40(7): 421-429.
14 Jocob A, Thomas R, Koshi SP, Andrew B, Muliyli J. Prevalence of primary glaucoma in an urban south Indian population. Ind J Ophthalmol 1998; 46: 81-86.

15 Dandona L, Dandona R, Mandal P, Srinivas M, John RK, McCarty CA et al. Angle closure glaucoma in an urban population in southern India. The Andhra Pradesh eye disease study. Ophthalmology 2000; 107(9): 1710-1716.

16 Rosenberg B. Studies in the depth of anterior chamber of the eye in primary glaucoma. Arch Ophthalmol 1950; 44(4): 523-538.

17 Spaeth GL. Gonioscopy. Uses old and new. The inheritance of occludable angle. Ophthalmology 1978; 85: 222-232.

18 Alsbirk PH. Anterior chamber depth and primary angle-closure glaucoma. II. A genetic study. Acta Ophthalmol (Copenhagen) 1975; 53(3): 436-449.

19 Lowe F. Causes of shallow anterior chamber in primary angle closure glaucoma. Am J Ophthalmol 1969; 67: 87-93.

20 Torniquist R. Shallow anterior chamber in acute glaucomas. Acta Ophthalmol 1953; 31: 1-74.

21 George R, Paul PG, Baskaran M, Ramesh SV, Raju P, Arvind $\mathrm{H}$ et al. Ocular Biometry in occludable angles and angle closure glaucoma: a population based survey. $\mathrm{Br} \mathrm{J}$ Ophthalomol 2003; 87(4): 399-402.

22 Congdon N, Wang F, Tielsch JM. Issues in the epidemiology and population based screening of primary angle closure glaucoma. Surv Ophthalmol 1992; 36: 411-423.

23 Grieten J, Weekers R. Etide des dimensions de la chamber anterieure de l'oeil humaine. III. Dans le glaucoma a angle ferme et dansle glaucoma a angle ouvert. Ophthalmologica (Basel) 1962; 143: 409-422. 